\title{
Applications of Rat Grimace Scale Method in Postoperative Pain Management in Rats
}

\author{
Alexandra DREANCĂ, Lucia BEL, Bogdan SEVASTRE*, Ioan MARCUS \\ Faculty of Veterinary Medicine, University of Agricultural Science and Veterinary Medicine, Manastur \\ Street. 3-5, 400372, Cluj-Napoca, Romania \\ * corresponding author: bogdan.sevaste@usamvcluj.ro
}

Bulletin UASVM Veterinary Medicine 74(1) / 2017,

Print ISSN 1843-5270; Electronic ISSN 1843-5378

DOI:10.15835/buasvmcn-vm: 12391

\begin{abstract}
Nowadays, 3R (replacement, reduction, refinement) concept lies in the core of laboratory animals science, one of the most important refinement strategies being the pain control. Rat Grimace Scale (RGS) is an actual and effective method used to assess pain in laboratory animals. The purpose of this study was to find the most effective post surgery analgesic protocol, by grading the changes in the animal's facial expression. In the present study we used 5 Wistar rats, one as control and four animals subjected to ovariectomy. Tramadol was administrated subcutaneously as it follows: 1 rat in pre-surgery dose of $25 \mathrm{mg} / \mathrm{kg}, 2$ rats in post-surgery a dose of $25 \mathrm{mg} / \mathrm{kg}$ and respectively $50 \mathrm{mg} / \mathrm{kg}$. The evaluation of pain was done across at the time $0,2,4$ and 6 hours post-surgery using the scores of 0, 1 and 2, 0 representing the absence of pain, 1 a moderate level of pain and 2 a high level of pain. The results of the study revealed that the spayed rat without Tramadol presented the highest level of pain (1.25 - 1.75). The pre-surgery analgesia enhanced the narcosis effect, but analgesia had a short term effect, with a moderate to high level of pain (1 - 1.5). To the animal treated with a dose of $25 \mathrm{mg} / \mathrm{kg}$ post-surgery, a moderate pain level was observed $(0.5-1.25)$ and to the animal with the highest dose an optimal analgesia was determined $(0.25)$. The expected analgesic effect was put forward by the $50 \mathrm{mg} / \mathrm{kg}$ dose of Tramadol and the RGS method was found to be suitable for the assessment of animal suffering in surgical experimental procedures.
\end{abstract}

Keywords: refinement, pain control, pain facial recognition

\section{INTRODUCTION}

"If we accept that animals can experience pain then we have an obligation to develop effective methods of recognizing it and assessing its nature and severity" (Leah, 2012). Nowadays, 3R (replacement, reduction, refinement) concept lies in the core of laboratory animals science. When replacement is not possible the scientist have the moral and legal task to reduce at minimum the pain and distress in animals subjected to experimental procedures. Only if we are able to quantify pain, then we can effectively asses the severity of experimental procedures or to find the proper analgesic therapy. Traditionally pain assessment was done by recording of physiological changes and latter by behavioral observation, both methods being limited by the fact that physiological parameter (such as heart rate or respiratory rate) or behavioral changes do not reflect satisfactory the intensity of pain (Mogil et al., 2010). First developed for non speaking humans as infants, patients with dementia, face grimace scale was recently validated for the pain assessment in rats, mice and rabbits (Asgar, 2015 ). It is long time known that facial expression is associated with pain (Leach, 2012). The recent development of rodent grimace scales has expanded the ability to assess pain in rodents and detect failures in translational research (De Rantere et al., 2016). The Rat Grimace Scale (RGS) consists of four 
facial action units: orbital tightening, nose/cheek appearance, ear and whisker position, which are scored using images by an observer. The RGS is used mainly to observe and diagnose spontaneous pain.

There are two methods of RGS scoring: imaging scoring, the classical method (Sotocinal et al., 2011), and real-time scoring (Leung et al., 2016). In a study on these two methods, Leung et al. (2016) said that the real-time application of RGS is better because it allows early identification of pain, facilitates timely intervention, improving the welfare of the animals.

Pain control is widely accepted as the only humanly acceptable method to perform surgery. However, each year, millions of rats undergo surgery for research purposes, but only $20 \%$ of researches worldwide reported the administration of analgesics after surgery. The treatment of pain itself varies (Roughan et al., 2004), drug and dosage varies from institution to institution without a scientific assessment of the analgesic efficiency (Stokes, 2009).

In the present study, we provide further evidence for using the RGS as a reliable method to assess the optimal analgesic protocol, in postoperative pain control. Hence, RGS is used to identify the optimal dose of Tramadol, an opioid analgesic, in both preoperative and postsurgical administration.

\section{MATERIALS AND METHODS}

The experiment was conducted on 5 healthy, adult, female Wistar rats. The procedures involving the present project were conducted following the guidelines of Directive 2010/63/EU and national low $43 / 2015$. The Wistar rats were housed in a facility under standard conditions: temperature $23^{\circ} \mathrm{C}$, humidity $55 \%$, light/dark cycle $12 / 12$. The animals were fed on standard rodent granular food and they had unrestricted access to water.

As a pain model, ovariectomy was performed on 4 of the rats. The surgery was performed under general anesthesia with isoflurane/oxygen. The induction protocol was performed with Xylazine and Ketamine. Following shaving and disinfection an abdominal laparatomy was performed, with the removal of both ovaries. Muscle and skin layers were closed with polydioxanone suture.

Tramadol was administrated subcuta-neously as it follows: 1 pre-surgery dose of $25 \mathrm{mg} / \mathrm{kg}$, 2 post-surgery a dose of $25 \mathrm{mg} / \mathrm{kg}$ and a $50 \mathrm{mg} / \mathrm{kg}$ dose. The dosage, route and administration time were established according to the previous studies (Cannon et al., 2010). The evaluation of pain was done across at the time $0,2,4$ and 6 hours postsurgery.

RGS assessment. Postoperative pain intensity was measured using the classic method previously reported by Sotocinal et al. Rats were placed individually in a glass cage. Facial expression of rats were recorded by two video cameras (Samsung Galaxy S5) for 30 minutes for the control rat without surgery, and 10 minutes for the others across the time intervals mentioned above. Still frames (front view and lateral view) were captured using Windows Movie Maker to display each rat's grimace changes. The RGS was determined by three evaluators who were blind to the study treatment using a 3 - point scale (0-no pain, 1- moderate pain, 2- intense pain) for each facial action.

Statistics. All data are reported as the mean \pm SEM. To assume Gaussian distribution normality distribution was checked by ShapiroWilk normally test. The values were not normally distributed, thus multiple pare-wise comparison was performed by one-way analysis of variance Kruskal-Walis, followed by post test Dunns. Statistical significance was at $\mathrm{p}<0.05 \quad(95 \%$ confidence interval). Statistical values and figures were obtained using GraphPad Prism 5.0 software.

\section{RESULTS AND DISCUSSION}

The study showed that the untreated ovariectomised rat presented the most intense pain (1.5-2) (fig.1.A). Presurgical analgesia with $25 \mathrm{mg} / \mathrm{kg}$ enforced the narcosis effect, the subject (fig.1.B) being the last to wake up, but the analgesic effect was short-lived, with moderate to high pain levels (1-1.5). The subject treated with $25 \mathrm{mg} / \mathrm{kg}$ post-surgery (fig.1.C) showed moderate pain levels $(0.5-1.25)$. The subject treated with Tramadol $50 \mathrm{mg} / \mathrm{kg}$ post-surgery (fig.1.D) showed an optimal analgesic effect (0.25).

The orbital tightening the pain score was evaluated as follows: the ovariectomised rat without analgesics presented intense pain during the evaluation period (1.4-2) compared to the control rat $(0.0)(p<0.001)$. The rats who received analgesics showed moderate pain level during the evaluation period $(1-1.6)(\mathrm{p}<0.01)$ compared to 


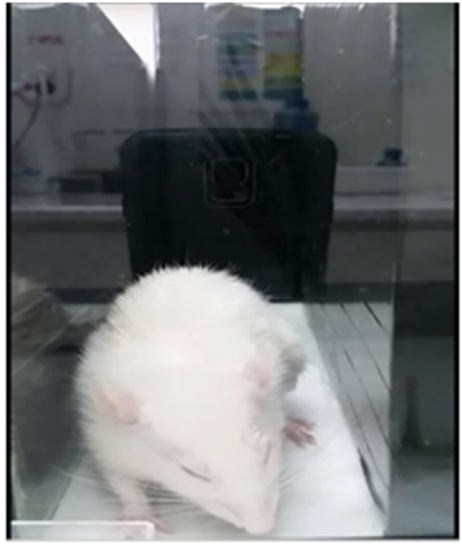

A

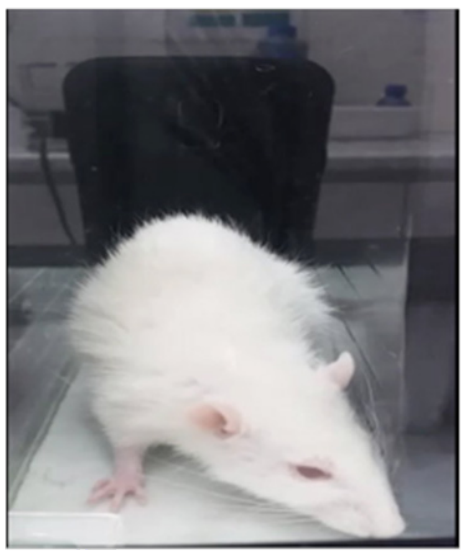

C

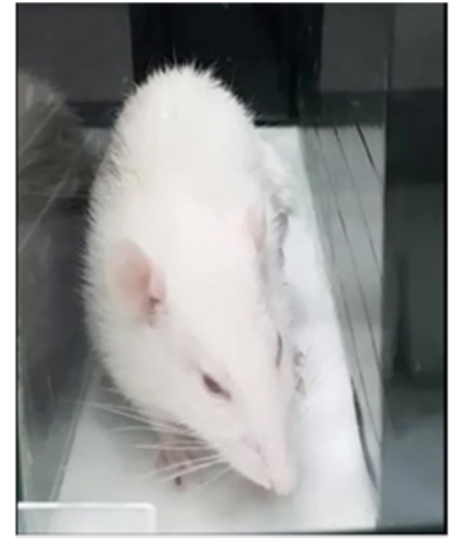

B

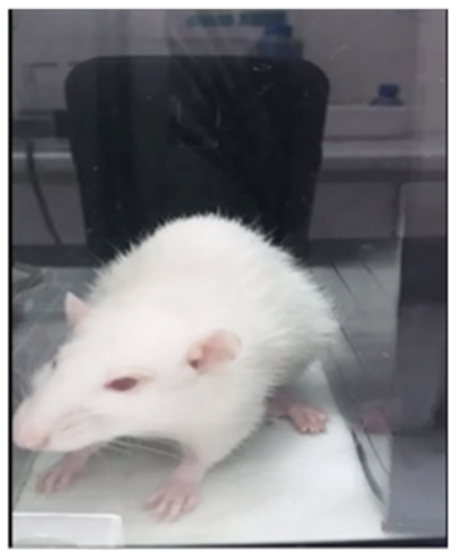

D

Fig. 1. Pain intensity in spayed rats regarding RGS; A - intense pain (2.0); B - moderate pain to high pain level (1.0 - 1.5); C - moderate pain (0.5-1.25); D - low or absent pain level (0.0-0.25).

the control, until the end of the time interval where in the two rats treated post surgical the absence of pain was observed (fig.2).

The nose/cheek flattening, pain intensity is increased in the rats treated with $25 \mathrm{mg} / \mathrm{kg}$ pre and post surgery and in the one without analgesics (2.0) only in the former part of the evaluation compared to control $(\mathrm{p}<0.01)$. During the final three periods the pain intensity level is moderate $(0.5-1.0)$ compared with control $(0.0)(\mathrm{p}<0.05)$.

The ear positions, pain levels are increased up to 2 hours post-surgery. Immediately after surgery, high pain intensity is present in untreated subjects, and those treated with $25 \mathrm{mg} / \mathrm{kg}$ and $50 \mathrm{mg} / \mathrm{kg}$ post-surgery (1.7), compared to the control. The subject treated with $25 \mathrm{mg} / \mathrm{kg}$ presurgery presented intense pain 2 hours postsurgery (1.7). During the rest of the evaluation periods, all subjects showed moderate pain (1.01.3) compared to the control.
The whisker positions, pain levels are significantly increased (2.0) immediately after surgery for the untreated rat $(p<0.001)$, later settling down to moderate-low levels for each subject $(0-1.0)(\mathrm{p}<0.05)$.

We are fully aware that the limited number of animals used in the present study is a serious limitation for a final recommendation regarding the dose of tramadol used as painkiller in postsurgical pain control. This is why we consider our data regarding the dose and time of administration as preliminary results. Our study aims to assess the value of RGS, as evaluation method in effectiveness of analgesic therapy. Tramadol is a narcotic-like pain reliever, it is used to treat moderate to severe pain (www.drugs.com). It is a common analgesic used in animal postsurgical treatment (Waite, 2015). To determine the efficiency of this drug for reducing spontaneous and acute pain after surgery Tramadol was administered in different dosage after and before the surgery. The study showed 


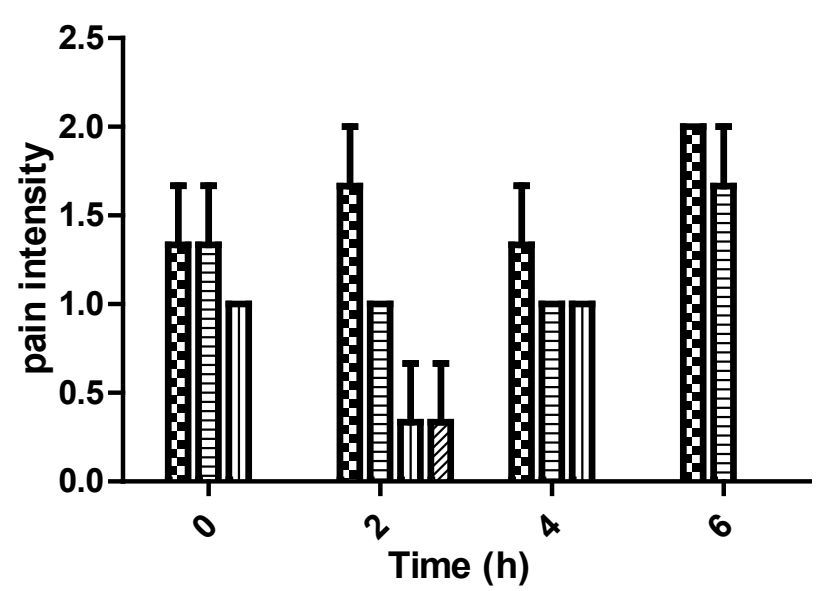

Control
OV
OV $25 \mathrm{mg} / \mathrm{kg}$ b.w. presurgery
OV $25 \mathrm{mg} / \mathrm{kg}$ b.w. postsurgery
OV $50 \mathrm{mg} / \mathrm{kg}$ b.w. postsurgery

Fig. 2. Pain levels according to orbital tightening

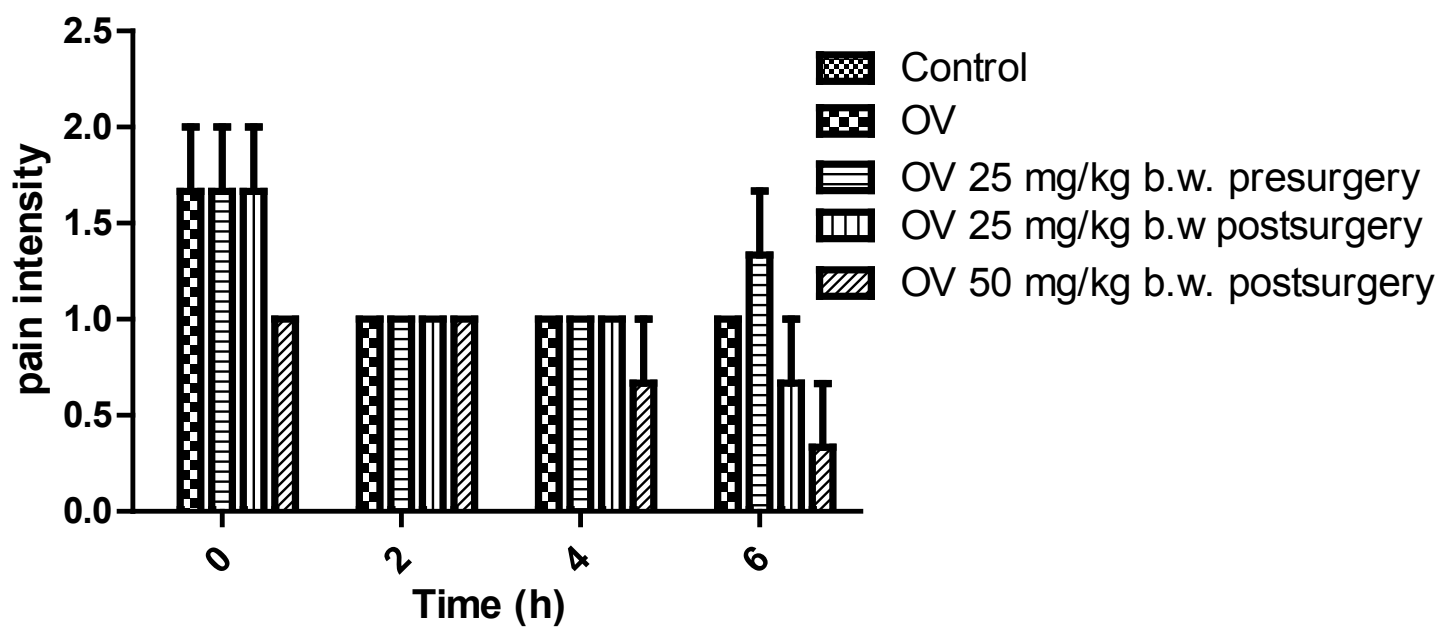

Fig. 3. Pain levels according to nose/cheek flattening

that administrated before the surgery, Tramadol enhanced the narcosis effect and reduced the pain level up to 2 hours after the surgery. But during the last 4 hours tested, the rat with presurgery analgesia experimented moderate to severe pain. In addition to animal welfare, pain must be minimized to avoid confounding experimental outcomes (Chi, 2015). Spontaneous pain is the most common complication after surgery (Stokes, 2009). Furthermore, we found that the minimum dosage of $25 \mathrm{mg} / \mathrm{b} . \mathrm{w}$ is not enough to alleviate pain. The rat treated with this dosage experimented moderate to severe pain during the experiment. In the final time interval the pain intensity began to decrease. Our current data supports the fact that only the subject treated with Tramadole $50 \mathrm{mg} / \mathrm{b} . \mathrm{w}$ post-surgery showed an optimal analgesic effect. This discrepancy between the doses used for pain treatment may be the result of conflicting or variable recommendations between institutions or due to limited empirical research on which recommendations are based (Waite, 2015). In our point of view, to clearly assess the dose and administration protocol for Tramadole in rats, further research is needed, in terms of using a larger, statistically significant number of animals per group, and simultaneously using of other methods of pain assessment.

Rat Grimace Scale is a reliable method of assessing spontaneous pain in laboratory rats that is available to all researchers and animal care staff. This method has been recently validated for pain assessment. The standard method along with the real-time application of the rat Grimace Scale could reliably and accurately improve research and hundreds of experimental models, in order to eliminate the pain factor, so it does not interfere with the final purpose of the experiment. This 


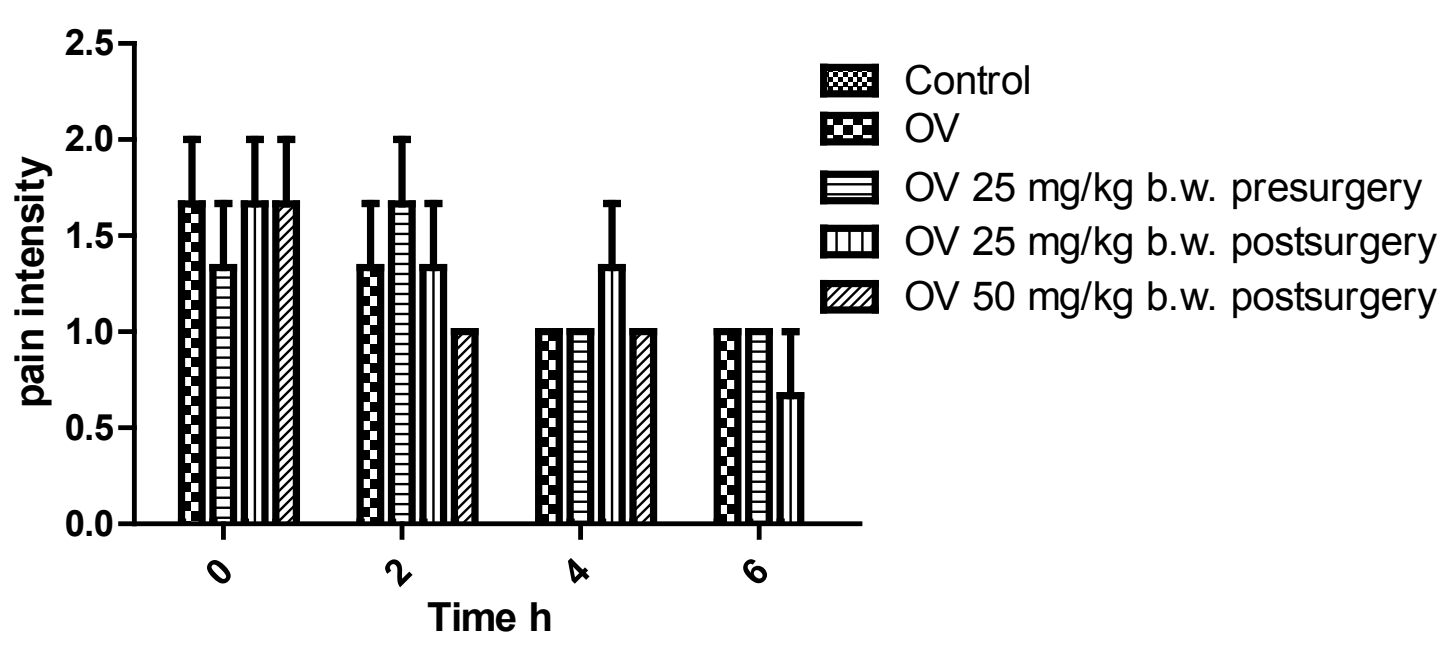

Fig. 4. Pain levels according to ear position

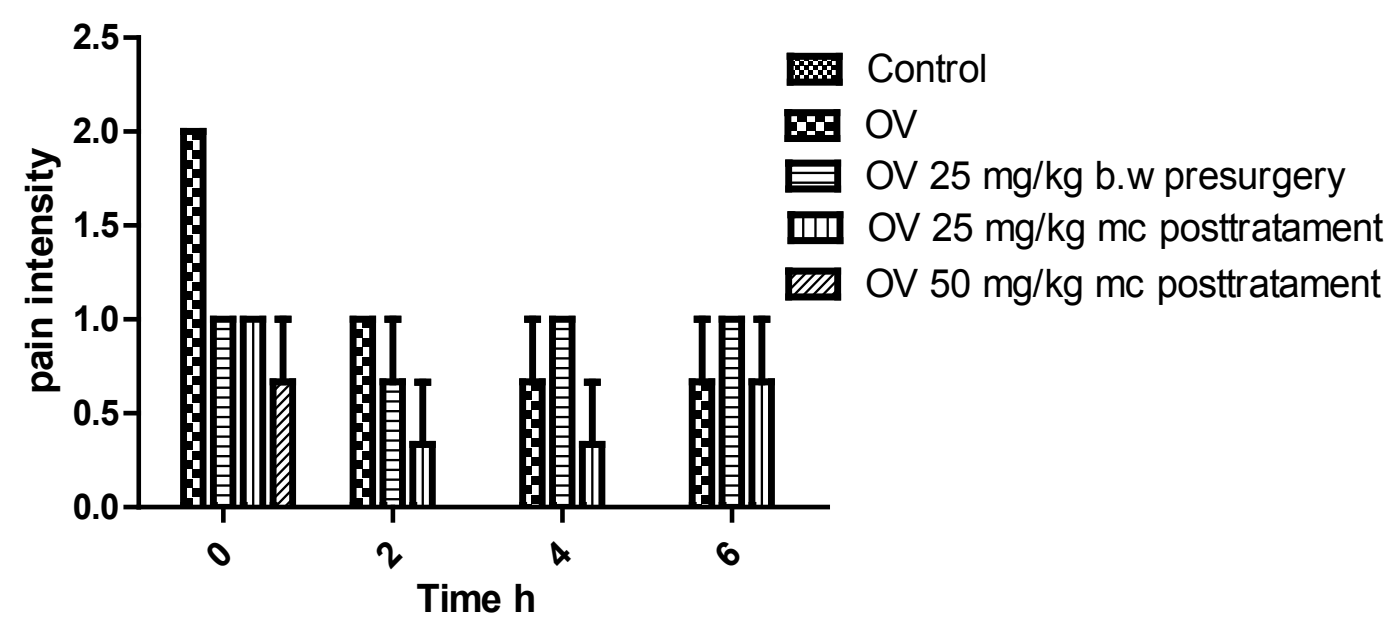

Fig. 5. Pain levels according to whisker position

method is allowing refinement of laboratory rat welfare through rapid identification of pain and early intervention (Sotocinal, 2011).

\section{CONCLUSIONS}

Our study clearly showed that RGS is a reliable protocol for pain assessment in experimental surgery. The present preliminary findings indicate that the proper analgesic effect was encountered in the animal injected subcutaneously with 50 $\mathrm{mg} / \mathrm{b} . \mathrm{w}$ Tramadol, after the surgery.

\section{REFERENCES}

1. Asgar J, Zhang Y, Saloman JL, Wang S, Chung MK, Ro JY (2015). The Role of TRPA1 in Muscle Pain and Mechanical Hypersensitivity under Inflammatory Conditions In Rats. NeuroScience 310: 206-215.

2. Chi H, Kawano T, Tamura T, Iwata H, Takahashi $Y$, Eguchi S, Yamazaki F, Kumagai N, Yokoyama M (2013).
Postoperative Pain inpairs subsequent performance on a spetial memory task via effects on N-methyl-D-aspartat receptor in aged rats. Life Sciences 93: 986-993.

3. Cannon CZ, Kissling GE, Hoenerhoff MJ, King-Herbert AP, Blankenship-Paris T (2010). Evaluation of dosages and routes of administration of tramadol analgesia in rats using hot-plate and tail-flick tests. Lab Anim (NY). 39(11): 342-51.

4. De Rantere D, Schuster CJ, Reimer JN, Pang DS (2016). The relationship between the Rat Grimace Scale and mechacanical hypersensitivity testing in three experimental models. European Journal of Pain 20:417426.

5. Leach M (2012). Pain assessment in laboratory animals using behaviour and facial expression. Workshop on Pain Assessment and Alleviation, Newcastle University.

6. Leung V, Zhang E, Pang DSJ (2016). Real-time application of the Rat Grimace Scale as a welfare refinement in laboratory rats. Nature Scientific Reports 6:31667.

7. Mogil JS, Davis KD, Derbyshire SW. (2010). The necessity of animal models in pain research. Pain 151: 12-17. 
8. Olivier V, Rantere D, Ritchie R, Chisholm J, Hecker K, Pang DSJ (2014). Psychometric Assessment of the Rat Grimace Scale and Development of an Analgesic Intervention Score. Plos One 9(5): 1-7.

9. Roughan JV, Flecknell PA (2004). Behaviour-based assessment of the duration of the duration of laparotomyinduced abdominal pain and the analgesic effect of caprofen and buprenorphine in rats. Behav Pharmacol 15:461-472.

10. Roughan JV, Flecknell PA, Davies BR (2004). Behavioural assessment of the effects of tumour growth in rats and the influence of the analgesics caprofen and meloxicam. Laboratory Animals 38:286-296.

11. Sotocinal SG, Sorge RE, Zaloum A, Tuttle AH, Martin LJ, Wieskopf JS, Mapplebeck JCS, Wei P, Zhan S, Zhang S, McDougall JJ, King OD, and Mogil JS, Rat Grimace Scale (RGS) The Manual, Molecular Pain, in press.

12. Sotocinal SG, Sorge RE, Zaloum A, Tuttle AH., Martin LJ, Wieskopf JS, Mapplebeck, JCS, Wei P, Zhan S, Zhang S,
McDougall JJ, King OD, and MogilJS (2011). The Rat Grimace Scale: a partially automated method for quantifying pain in the laboratory rat via facial expressions. Molecular Pain 7:55.

13. Stokes EL, Flecknell PA, Richardson CA (2009). Reported analgesic and anaesthesic administration to rodents undergoing experimental surgical procedures. Laboratory Animals 43:149-154.

14. Waite ME, Tomkovich A, Quinn TL, Schumann AP, Dewberry LS, Totsch SK, Sorge RE (2015). Efficacy of Common Analgesiscs for Postsurgical Pain in Rats. Journal of the American Association for Laboratory Animal Science 54(4): 420-425.

15. www.digires.co.uk

16. www.ahwla.org.uk

17. www.nc3rs.org.uk

18. www.drugs.com 